\title{
Survival Analysis of Premature Infants Admitted to Neonatal Intensive Care Unit (NICU) in Northwest Ethiopia using Semi-Parametric Frailty Model
}

\author{
Sheferaw Yehuala', SalieAyalew ${ }^{2}$ and Zinabu Teka ${ }^{2 *}$ \\ ${ }^{1}$ Department of Statistics, College of Natural Sciences, Jimma University, Ethiopia \\ ${ }^{2}$ Department of Statistics, College of Natural and Computational Science, University of Gondar, Ethiopia
}

\begin{abstract}
In this research, the cox proportional hazard model and the semi-parametric gamma frailty model were compared on the survival of premature infants admitted to neonatal intensive care unit from December 29, 2011 to April 6, 2014. A retrospective study design was used to collect the data from patients chart. A frailty effect $(\theta=0.252, P$-Value $=0.0031$ $<\alpha=0.05$ ) was obtained from the semi-parametric gamma frailty model, and mortality was depend within and across categories of premature infants based on their gestational age. The values of frailty were dispersed and hence induce greater heterogeneity in the infant hazards. Therefore, when there is heterogeneity, semi-parametric gamma frailty model could be used and lead to acceptable conclusions. Both models identifies Antenatal Care Visit, gravidity of (6-10), HIV status of mother, Respiratory Distress Syndrome, Prenatal Asphyxia, anemia and breastfeed initiated as the most determinant and statistically associated with time to death of premature infants admitted to NICU. Based on the model comparison analysis, semi-parametric gamma frailty was the best model to fit the data.
\end{abstract}

Keywords: Cox proportional hazard model; Frailty model; Premature infant; Hospital; Neonatal mortality

\section{Introduction}

Infants born before 37 completed weeks of gestation are known as premature or preterm infants. Premature infants come early into the world. They are born fragile, small and weighing less than full term infants. Preterm birth refers to the birth of a baby that occurs before 37 completed weeks of gestation. It can be further sub categorized as late preterm delivery from 34 to 36 , moderately preterm from 32 to 34 , very preterm less than 32 , and extremely preterm less than 28 weeks of gestation WHO [1] and Offiah et al. [2]. Infants born with in preterm category share similar risk for death, weight, size and strength, are still at higher risk for health and developmental problems compared to those infants born full term [3]. Preterm birth can also be defined by birth weight: low birth weight less than $2500 \mathrm{~g}$, very low birth weight $1500 \mathrm{~g}$, and extremely low birth weight less than 1000 [2]. Premature birth is the major cause of prenatal morbidity and mortality all over the world [4].

Globally, an estimated 13 million infants are born before 37 completed weeks of gestation annually. Rates are generally highest in low and middle income countries and increasing in some middle and high income countries [5]. More than 1 in 10 of the world's babies born in 2010 were born prematurely, making an estimated 15 million preterm births, of which more than 1 million died as a result of their prematurity $[6,7]$. Preterm are now the second leading cause of death in children less than 5 years and the single most important cause of death in the critical first month of life. Preterm birth accounts for 3.1\% of all Disability Adjusted Life Years (DALYs) in the Global Burden of Disease, more than for HIV and malaria [1]. In Ethiopia, According to report of UNICEF [8], one of the main causes of neonatal death is preterm birth accounts for $23 \%$ of all other causes of neonatal death. Given the frequency of preterm birth worldwide, it is likely that most people will experience the tragedy of preterm birth at some point in their lives, either in family members or indirectly through friends.

Preterm birth has multiple factors; therefore solutions will not come through a single discovery but rather from an array of discoveries addressing multiple biological, clinical, and social behavioral risk factors. Therefore, Survival analysis consists in determining study subjects survival when exposed to the variables considered risk factors. It is currently known that the study of risk factors for infant mortality is very important, as, particularly in the newborn, it can be considered one of the best quality indicators for health care, as well as an indicator for population social and economic welfare [9].

Frailty models account for unobserved heterogeneity that occurs because some observations are more prone to failure and therefore frail than others in a data set. Therefore, the objective is to introduce an additional parameter to the hazard rate that accounts for the random frailties. These frailties can be specific to individuals or groups, and are referred to as individual frailty or shared frailty respectively.

The shared frailty model is relevant to event times of related individuals, similar organs and repeated measurements. Individuals in a cluster are assumed to share the same frailty, which is why this model is called shared frailty model. It was introduced by [10] and extensively studied in [11]. Therefore, in this study, there may be similar frailty with in subcategories of preterm birth, based on weeks of gestational age. Infants are born with in preterm category share similar risk for death, weight, size and strength [3].

This study presents an extension of Cox model to frailty model in which the Cox-proportional of baseline hazard and the gamma distribution is used as frailty distribution. The gamma distribution is

*Corresponding author: Zinabu Teka, Department of Statistics, College of Natural and Computational Science, University of Gondar, Ethiopia, E-mail: take.z70@gmail.com

Received February 17, 2015; Accepted May 14, 2015; Published May 21, 2015

Citation: Yehuala S, Ayalew S, Teka Z (2015) Survival Analysis of Premature Infants Admitted to Neonatal Intensive Care Unit (NICU) in Northwest Ethiopia using Semi-Parametric Frailty Model. J Biomet Biostat 6: 223. doi:10.4172/21556180.1000223

Copyright: $\odot 2015$ Yehuala S, et al. This is an open-access article distributed under the terms of the Creative Commons Attribution License, which permits unrestricted use, distribution, and reproduction in any medium, provided the original author and source are credited. 
one of the most commonly used distributions for frailty. It has a closed form likelihood function that can be readily maximized. In addition, we study the performance of the Cox-proportional hazard model and compare with the semi parametric gamma frailty model by using AIC, BIC, I-likelihood, R2 and concordance probability.

\section{Method of Data Analysis}

\section{Data}

All medical records of preterm infants those who were admitted to NICU at University of Gondar Hospital from December 29, 2011 to April 6, 2014 were retrospectively reviewed by medical professionals.

\section{Survival data analysis}

The term "survival analysis" pertains to a statistical approach designed to take into account the amount of time an experimental unit contributes to a study. That is, it is the study of time between entry into observation and a subsequent event. Originally, the event of interest was death hence the term, "survival analysis" is given. The analysis consisted of following the subject until death occurs considered as event of interest. Applications now include time until onset of disease, stock market crash, equipment failure, earthquake, and so on. The best way to define such events is simply to realize that these events are a transition from one discrete state to another at an instantaneous moment in time. Of course, the term "instantaneous", which may be years, months, days, minutes, or seconds, is relative and has only the boundaries set by the researcher.

\section{The cumulative distribution function}

The Cumulative Distribution Function (CDF) is very useful in describing the continuous probability distribution of a random variable, such as time, in a survival analysis. The CDF of a random variable $\mathrm{T}$, denoted $F T(t)=P T(T \leq t)$. This is interpreted as a function that will give the probability that the variable $\mathrm{T}$ will be less than or equal to any value $t$ that we choose. Several properties of a distribution function $F(t)$ can be listed as a consequence of the knowledge of probabilities. Because $F(t)$ has the probability $0 \leq F(t) \leq 1$, then $F(t)$ is a non-decreasing function of $t$, and as $t$ approaches positive infinity, $\mathrm{F}(\mathrm{t})$ approaches 1 . It is important to estimate the median survival time at $F-1 T(0.5)$.

\section{The survival function}

Let the random variable $T \geq 0$ have a PDF $F(t)$ and $\operatorname{CDF} F(t)$. Then the survival function takes on the following form:

$$
S(t)=P(T>t)=1-F(t)
$$

That is, the survival function gives the probability of surviving or being event free beyond time $t$. Because $S(t)$ is a probability, it is positive and ranges from 0 to 1 . It is defined as $S(0)=1$ and as $t$ approaches positive infinity, $\mathrm{S}(\mathrm{t})$ approaches 0 .

\section{The hazard function}

The Hazard Function $\mathrm{h}(\mathrm{t})$ is given by the following:

$$
h(t)=P[t<T\langle(t+\Delta) / T\rangle t)]=\frac{f(t)}{1-F(t)}=\frac{f(t)}{S(t)}
$$

The hazard function describes the concept of the risk of an outcome (e.g., death, failure, hospitalization) in an interval after time $t$, conditional on the subject having survived to time t. It is the probability that an individual dies somewhere between $t$ and $t+\Delta$, divided by the probability that the individual survived beyond time t. The hazard function seems to be more intuitive to use in survival analysis than the PDF because it attempts to quantify the instantaneous risk that an event will take place at time $t$ given that the subject survived to time $\mathrm{t}$. The cumulative hazard function $\mathrm{H}(\mathrm{t})$ is the integral of the hazard function between integration limits of 0 and $t$.

\section{Cox proportional hazard model}

Cox proportional hazard model is usually written in terms of the hazard model formula. This model gives an expression for the hazard at time $t$ for an individual with a given specification of a set of explanatory variables denoted by $\mathrm{X}$ and it is generally given by:

$$
h\left(t, X_{i}, \beta\right)=h_{o}(t) \exp \left(\beta^{\prime} X_{i}\right)
$$

where $h_{o}(t)$ is the baseline hazard function which is obtained all X's are set to zero, $\mathrm{Xi}$ is the vector of values of the explanatory variables for the ith individual at time $\mathrm{t}$ and $\beta=\left(\beta_{1}, \beta_{2}, \ldots, \beta_{k}\right) T$ is the vector of unknown regression parameters that are assumed to be the same for all individuals in the study, which measures the influence of the covariate on the survival experience. An attractive property of the Cox model is that, even though the baseline hazard part of the model is unspecified, it is still possible to estimate the $\beta$ 's in the exponential part of the model. So, it can equally be regarded as linear model, as a linear combination of the covariates for the logarithm transformation of the hazard ratio given by:

$$
\log \left\{\frac{h\left(t, X_{i}, \beta\right)}{h_{o}(t)}\right\}=\beta^{\prime} X(3.7)
$$

The cumulative hazard function is given by:

$$
H(t)=\mathrm{H}_{\mathrm{o}}(\mathrm{t}) \exp \left(\beta^{\prime} X\right)
$$

From model (3.6), we obtained the survivor function given by:

$$
S\left(t, X_{i}, \beta\right)=\left[S_{o}(t)\right]^{\exp \left(\beta^{\prime} X\right)}
$$

Where: $S_{0}(t)$ is a baseline survival function.

\section{Semiparametric frailty models}

In frailty models, the variability of survival times can be divided into two parts. One part is observed risk factors, known as covariates, and the other part is unobserved risk factors, known as frailty. The Univariate frailty model presents the population as a mixture in which baseline hazard is common to all individuals but each individual has his/her own frailty. Suppose we have a sample of $j$ observations in a study. Some of these observations fail earlier than others due to unobserved heterogeneity. The proportional hazards model assumes that conditional on the frailty, the hazard function for an individual at time $\mathrm{t}>0$ is

$$
h_{j}\left(t, X_{j}, \beta\right)=h_{o}(t) \exp \left(\beta^{\prime} X_{j}+W_{j} \phi\right), j=1,2, \ldots, n
$$

Where, $W_{\text {is }}$ a frailty term from a probability distribution with a mean of 0 and variance of 1 . If $W_{j}$ could be measured and included in the model, then $\phi$ would go to 0 and we would obtain the standard proportional hazards model. The hazard function conditional on both covariates and frailty can be rewritten as:

$$
h_{j}\left(t, X_{j}, \beta\right)=h_{o}(t) U_{j} \exp \left(\beta^{\prime} X_{j}\right), j=1,2, \ldots, n
$$

Where, $U_{j}=\exp \left(W_{j} \phi\right)$.This shows that the hazard of an individual also depends on an unobservable random variable, $U$, which acts multiplicatively on the hazard rate. If frailty is not taken into account, then $U_{j}=1$. 
In the Univariate case, frailty models are used to make adjustments for over dispersion. When unobserved or unmeasured effects are ignored, the estimates of survival may be misleading. Therefore, correction for this over dispersion is needed in order to allow for adjustments for those important frailties [12].

Shared frailty model is similar to the individual frailty model except the only difference is that frailty is now shared among the $n_{i}$ observations in the $i^{\text {th }}$ group. Suppose we have $j$ observations and $i$ subgroups. Each subgroup consists of $n_{i}$ observations and $\sum_{i=0}^{G} n i=n$, where $\mathrm{n}$ is the total sample size. The hazard rate for the $j^{\text {th }}$ individual in the $i^{\text {th }}$ subgroup is given by:

$$
h_{i j}\left(t, X_{i j}, \beta\right)=h_{o}(t) \exp \left(\beta^{\prime} X_{i j}+W_{j} \phi\right), i=1, \ldots, G, j=1,2, \ldots, n_{i}
$$

Where, $W_{i}$ are frailty terms for subgroups and their distribution is again assumed to be independent with a mean of 0 and a variance of 1. The hazard function conditional on covariates and frailties can be rewritten as:

$$
h_{i j}\left(t, X_{i j}, \beta\right)=h_{o}(t) U_{j} \exp \left(\beta^{\prime} X_{i j}\right), i=1, \ldots, G, j=1,2, \ldots, n_{i}
$$

Where, $U_{j}=\exp \left(W_{j} \phi\right)$

As in the proportional hazards model, parametric or nonparametric forms of baseline hazard can be assumed in frailty models. If non-parametric form is assumed for $h_{o}(t)$, then semi parametric proportional hazards model is considered and the estimates are usually obtained by using Expectation-Maximization (EM) algorithm. If parametric form for $h_{o}(t)$ is assumed, then maximum likelihood estimates can be obtained by maximizing the likelihood function. In this study, we are only considering the semi parametric forms of baseline hazard. Most applications assume Cox Proportional baseline hazard.

The basic idea of a frailty model is to incorporate an unmeasured "random" effect in the hazard function to account for heterogeneity in the subjects. When the observed data consist of triples $\left(t_{i}, x_{i}, c_{i}\right), i=1$, $2, \ldots, \mathrm{n}$ denoting the observed follow up times, the vector of $\mathrm{p}$ covariates, and a right censoring indicator variable, the hazard function at time $t$ for the $\mathrm{i}^{\text {th }}$ subject is, under the proportional hazards model,

$$
h\left(t, X_{i}, \beta\right)=h_{o}(t) \exp \left(\beta^{\prime} X_{i}\right)
$$

This idea extends to models with time varying covariates, with the usual change in notation.

A frailty model includes, in the hazard function, the value of an additional unmeasured covariate, the frailty, denoted $U_{i}$, yielding a hazard function:

$$
h_{f}\left(t, X_{i}, \beta\right)=U_{j} h\left(t, X_{i}, \beta\right)
$$

We use the subscript $f$ in equation (10) to represent a hazard function that has been modified by the inclusion of a frailty. An important statistical assumption is that the frailty is independent of any censoring that may take place. If the value of the frailty in (10) is greater than one, the subject has a larger than average hazard and is said to be more "frail". On the other hand, if the value of the frailty is less than one, the subject is less "frail" than an average subject. In particular, due to the fact the "most" frail individuals tend to fail early in the follow-up, the average hazard ratio tends to decrease over time $[11,13]$. Since the major thrust of this study is modeling with the proportional hazards model, we do not consider parametric models with frailties in any more detail. In the remainder of this section, we focus on adding a frailty to the semi parametric hazard model that is described in (10). In this study, we focus on the semi parametric gamma frailty distribution model.

\section{Gamma distribution}

Suppose a random variable $T>0$ is gamma distributed with scale parameter $\lambda>0$ and shape parameter $\alpha>0$, i.e. $T \sim \operatorname{Gamma}(\lambda, \alpha)$. The probability density function (PDF) of a random variable $\mathrm{T}$ is:

$$
f_{T}(t)=\frac{\lambda^{\alpha} t^{\alpha-1} e^{-\lambda t}}{\tilde{A}(\alpha)}, t>0
$$

Where, $\Gamma(k)=\iint^{k-1} e^{-s} d s$ is the Gamma distribution? The expected value and variance of the gamma distribution are as follows:

$$
\begin{aligned}
& E(T)=\frac{\alpha}{\lambda} \\
& \operatorname{Var}(T)=\frac{\alpha}{\lambda^{2}}
\end{aligned}
$$

The survival and hazard functions of the gamma distribution are given by:

$$
\begin{aligned}
& S(t)=\frac{\tilde{A}(\alpha, \lambda t)}{\tilde{A}(\alpha)} \\
& h(t)=\frac{\lambda^{\alpha}}{\tilde{A}(\alpha, \lambda t)} t^{\alpha-1} e^{-\lambda t}, t>0
\end{aligned}
$$

Where, $\Gamma(\alpha, \lambda t)$ is the upper incomplete gamma function.

The hazard function is decreasing, constant and increasing when 0 $<\alpha<1, \alpha=1$ and $\alpha>1$ respectively.

The gamma distribution is very well known and has simple densities. It is the most common distribution used for describing frailty. Even though gamma models do not have closed form expressions for survival and hazard functions, from a computational view, it fits well to frailty data and it is easy to derive the closed form expressions for unconditional survival and hazard functions. For this reason, this distribution is used often in most applications. Frailties appearing in the conditional likelihood can be integrated out and hence give simple expressions for marginal likelihood. Thus, it is easy to obtain parameter estimates by maximizing the marginal likelihood.

There are many applications of the gamma frailty model. Lancaster [14] suggested this model for the duration of unemployment. Andersen et al. used the gamma frailty model to check the proportional hazards assumptions in his study of malignant melanoma. Vaupel et al., [15] used the gamma distribution in their studies on population mortality data from Sweden. The gamma distribution has two advantages as a frailty distribution. First, the frailty distribution of the survivors at any given age is again a gamma distribution, with the same parameter and a different scale parameter. The second advantage is that the frailty distribution among the persons dying at any age is also a gamma distribution, with the same shape parameter plus one, and a scale parameter as a function of the age at death. However, there are no known biological reasons which make the gamma distribution preferable than other distributions.

In gamma frailty models, the restriction $\alpha=\lambda$ is used, which results in expectation of 1 .The variance of the frailty variable is then $1 / \lambda$. Assume that the frailty term $\mathrm{U}$ is distributed as gamma with $E(U)=1$ and $\operatorname{Var}(\mathrm{U})$ $=\theta$. Then $\lambda=\alpha=1 / \theta$. The distribution function of the frailty term $\mathrm{U}$ is then one parameter gamma distribution, $U_{i} \sim \operatorname{Gamma}(1 / \theta, 1 / \theta)$ :

$$
g(u)=\frac{u^{1 / \theta-1} \exp (-u / \theta)}{\tilde{A}(1 / \theta) \theta^{1 / \theta}}, \theta>0
$$

$U>1$ indicates that individuals in group $i$ are frail, whereas $U<1$ indicates that individuals are strong and have lower risk. 


\section{Model comparison methods}

Model comparison and selection are among the most common problems of statistical practice, with numerous procedures for choosing among a set of models proposed in the literature [16-21], for recent reviews. Most selection methods are defined in terms of an appropriate information criterion, a mechanism that uses data to give each candidate model a certain score; this then leads to a fully ranked list of candidate models, from the best to the worst. Here, we use these criterions to determine the best model between frailty model and Cox proportional hazard model. In order to compare proposed models we use Bayesian Information Criterion $(B I C)$, Akaike Information Criterion $(A I C)$ and Deviance Information Criterion (DIC).

$A I C$ providing a balance between models fit (via the log-likelihood) and model effective degree of freedom [22,23] advocated that, given a class of competing models for a data set, one choose the model that minimizes:

$$
A I C=D(\hat{\theta})+2 P
$$

Where, $p$ represents the number of parameters of the model. $D(\hat{\theta})$ Represents an estimate of the deviance evaluated at the posterior mean, $\hat{\theta}=E(\theta /$ data $)$. The deviance is defined by, $D(\theta)=-2 \log L(\theta)$ where $\theta$ is a vector of unknown parameters of the model and $L(\theta)$ is the likelihood function of the model.

The AIC penalizes the number of parameters less strongly than the Bayesian information criterion (BIC), which was independently developed by [24-26] also have shown that the AIC tends to overestimate the number of parameters needed, even asymptotically. The Schwarz criterion indicates that the model with the highest posterior probability is the one that minimizes:

$$
B I C=D(\hat{\theta})+P \cdot \log (n)
$$

The major benefit of the BIC approximation is that it includes the BIC penalty for the number of parameters being estimated. The model with the smallest BIC value is chosen as the best model.

\section{Results and Discussions}

During the study period, 485 premature infants were admitted to the NICU of UOGH. Of these infants included in the analysis, 363 (74.8\%) infants were discharged (Improved, No change and Referred) at the end of the follow up and 122(25.2\%) infants were died. In this study, the overall mean ( $95 \% \mathrm{CI}$ ) and median length of hospital stay was $20.383(19.170,21.59)$ and 28 days, respectively (Table 1$)$.

Table 2 shows demographic and obstetrics factors by the premature infants' death status, mean and median $(95 \% \mathrm{CI})$ at the end of follow up. Among premature infants included in the analysis, $268(55.3 \%)$ were males and $217(44.7 \%)$ were females with mean (95\% CI) length of hospital stay $19.88(18.230,21.536)$ and $20.95(19.165,22.739)$ days respectively. The results of this table indicated that majority of 410 infants (84.5\%) of their mother had ANC follow up. The highest mean (95\% CI) length of hospital stay was $25.09(23.572,26.608)$ days for infants of gestational age $(32,34] ; 24.29(22.727,25.867)$ days for infants of Gestational age $(34,37)$ and the smallest mean $(95 \% \mathrm{CI})$ and median $(95 \% \mathrm{CI})$ length of hospital stay was $3.686(2.744,4.628)$ and $2.00(1.226$, 2.774) days for infants of Gestational Age $\leq 28$ respectively. Of the 122 who died, the highest number $92(19.0 \%)$ and $88(18.1 \%)$ premature infants were died from mother's parity of [1-5] and gravidity of [25], respectively. Out of 485 premature infants $291(60 \%)$ and $194(40 \%)$ were delivered by SVD and CS, respectively. Other description of premature data is shown at Appendix (A). The most common problems identified at birth in premature infants were Hypothermia 274 (56.5), RDS 54(31.8), Breast Feeding 194(19.4) and Neonatal Sepsis 310(63.9) and with median $(95 \% \mathrm{CI})$ survival time of $15(6.724,23.276), 5(2.680$ $7.320), 3(2.454,3.546)$ and $28(. .$.$) days, respectively. The other common$ causes of admissions for premature infants were jaundice 61(12.6\%), prematurity of apnea 53(10.9\%), SGA 30(6.2), hypoglycemia $68(14 \%)$, PNA 63(13\%) and congenital malformation 5(1\%).

Appendix (B) shows preterm infants average length of days stay in hospital and percentage of death for each maternal clinical factor. 40(8.2\%) premature infants were born from HIV positive mothers with mean $(95 \% \mathrm{CI})$ and median length of hospital stay is $14.59(10.763$, $18.427)$ and 10 days, respectively. Eight (1.6\%) were IDM with mean $(95 \% \mathrm{CI})$ and median $(95 \% \mathrm{CI})$ length of hospital stay were 11.0(4.236, $17.764)$ and $3.00(0.000,14.642)$ days, respectively.

Using premature dataset, we obtain estimated Kaplan Meier survivor curve that is a decreasing step functions of survival function versus survival time, as illustrated Figure 1, rather than smooth curves. It gives the probability that the premature infants survival exceeds at the specified days. The probability of premature infants survives more than 28 days is 0.5 , as illustrated horizontal dot reference line in the figure. This layout showed that the probability of survival of premature infants is high in the first day, which relatively decreases as follow up time increases. During the first day of hospital stay, the maximum (93.4\%) probability of survival was observed with a standard error of 0.0113 , at the $10^{\text {th }}$ days of hospital stay the probability of survival of premature infants was $73.25 \%$ with a standard error of 0.0231 , from 19 to 25 days of hospital stay the probability of survival of premature infants was $65.33 \%$ with a standard error of 0.0343 and at the $28^{\text {th }}$ days of hospital stay the probability of survival of premature infant was $30.62 \%$ with a standard error of 0.2180 for the follow up period of time.

\section{Comparison of survivor function between two or more groups of premature infants}

Plots of the Kaplan Meier curves for each category of the covariates Neonatal Sex, Mode of Delivery, Birth weight of infants, respiratory distress and Parity are shown in Figures 2- 6 respectively. Notice that the Kaplan Meier curve for infants those who had Respiratory Distress, weight less than 1600 gram and more than one parity of their mother were consistently lower than the KM curve for infants those who had no RDS, weight of greater than or equal to 1600 and zero parity of their mother, respectively. Figures 4- 6 indicates that infants those who had no RDS, weight greater than or equal to 1600 and zero parity of their mother has better survival prognosis than to those corresponding categories. Moreover, as the length of hospital stay in days increases, the two curves appear to get farther apart, suggesting that the beneficial effects of infants those who have better survival prognosis over those haven't are greater the longer one stays in hospital. However, Figure 2 and 3 indicates Kaplan Meier curves for each category of the variable neonatal sex and mode of delivery are crossed each other, that means there is no survival difference. Most of the KM curves show there is a difference between different categories except the graph of covariate congenital malformation and TTN.

Table 3 describe how to evaluate whether or not overall Kaplan Meier curves for two or more categories of covariates are statistically

\begin{tabular}{|c|c|c|c|c|}
\hline & $\begin{array}{c}\text { No. of preterm } \\
\text { infants }\end{array}$ & Death (\%) & $\begin{array}{c}\text { Median } \\
{[95 \% \mathrm{Cl}]}\end{array}$ & Mean[95\% Cl] \\
\hline Total & 485 & $122(25.2)$ & $28[28,]$. & $20.383[19.170,21.59]$ \\
\hline
\end{tabular}

Table 1: Average survival time of premature infants. 
Citation: Yehuala S, Ayalew S, Teka Z (2015) Survival Analysis of Premature Infants Admitted to Neonatal Intensive Care Unit (NICU) in Northwest Ethiopia using Semi-Parametric Frailty Model. J Biomet Biostat 6: 223. doi:10.4172/2155-6180.1000223

Page 5 of 12

\begin{tabular}{|c|c|c|c|c|c|}
\hline & & & Died & Mean & Median \\
\hline Covariates & Categories & Total (\%) & Count(\%) & Estimate[95 \% Cl] & Estimate[95 \% Cl] \\
\hline \multirow{2}{*}{ Neonatal Sex } & Male & 268(55.3) & $73(15.1)$ & $19.88 \quad[18.230,21.536]$ & $28.0[.,]$. \\
\hline & Female & $217(44.7)$ & $49(10.1)$ & $20.95 \quad[19.165,22.739]$ &.$[.,]$. \\
\hline \multirow{2}{*}{ Mode of delivery } & SVD & 291(60.0) & $79(16.3)$ & $19.82[18.240,21.395]$ & $28.0[.,]$. \\
\hline & CS & 194(40.0) & $43(8.9)$ & $21.26 \quad[19.429,23.096]$ &.$[.,]$. \\
\hline \multirow{2}{*}{ Antenatal Care Visit(ANC) } & No & $75(15.5)$ & $34(7.0)$ & $14.77[11.751,17.784]$ & $12.0[7.201,16.799]$ \\
\hline & Yes & $410(84.5)$ & $88(18.1)$ & $21.41[20.123,22.694]$ & $28.0[25.195,30.81]$ \\
\hline \multirow{3}{*}{ Gravidity } & 1 & $123(25.4)$ & $17(3.5)$ & $23.71[21.601,25.822]$ & $28.0[7.114,48.886]$ \\
\hline & $5-F e b$ & $310(63.9)$ & $88(18.1)$ & $18.69[17.185,20.187]$ & $26.0[.,]$. \\
\hline & 10-Jun & $52(10.7)$ & $17(3.5)$ & $18.00[14.878,21.122]$ &.$[.,]$. \\
\hline \multirow{3}{*}{ Parity } & 0 & $124(25.6)$ & $17(3.5)$ & $23.78 \quad[21.708,25.851]$ & $28.0 \quad[7.119,48.881]$ \\
\hline & 5-Jan & $325(67.0)$ & $92(19.0)$ & $18.78[17.333,20.227]$ &.$[.,]$. \\
\hline & 10-Jun & $36(7.4)$ & $13(2.7)$ & $15.98 \quad[12.489,19.471]$ & {$[.,]$.} \\
\hline \multirow{2}{*}{ Weight of Infants } & $<1600$ & $123(25.4)$ & $62(12.8)$ & $15.73[13.519,17.935]$ & $14.0[1.163,26.837]$ \\
\hline & {$[1600,2500]$} & $362(74.6)$ & $60(12.4)$ & $20.97 \quad[19.692,22.249]$ &.$[.,]$. \\
\hline \multirow{5}{*}{ Gestational Age } & $\leq 28$ & $51(10.5)$ & $51(10.5)$ & $3.686 \quad[2.744,4.628]$ & $2.00[1.226,2.774]$ \\
\hline & $(28,30]$ & $26(5.4)$ & $7(1.4)$ & $19.60[15.558,23.637]$ & .[. , .] \\
\hline & $(30,32]$ & $88(18.1)$ & $42(8.7)$ & $15.77 \quad[13.155,18.377]$ & $13.0[4.759,21.241]$ \\
\hline & $(32,34]$ & 142(29.3) & 15(3.1) & $25.09 \quad[23.572,26.608]$ & $28.0[.,]$. \\
\hline & $(34,37)$ & $178(36.7)$ & $7(1.4)$ & $24.29 \quad[22.727,25.867]$ &.$[.,]$. \\
\hline \multirow{2}{*}{ Multiple Pregnancy } & No & 294(60.6) & $58(12.0)$ & $21.63[20.070,23.188]$ & $28.0[15.466,40.53]$ \\
\hline & Yes & 191(39.4) & $64(13.2)$ & $18.03[16.220,19.838]$ &.$[.,]$. \\
\hline
\end{tabular}

Table 2: Average and $95 \% \mathrm{Cl}$ for average length of day stay in hospital, Frequencies and Percentages death for Demographic and Obstetrics Factors.

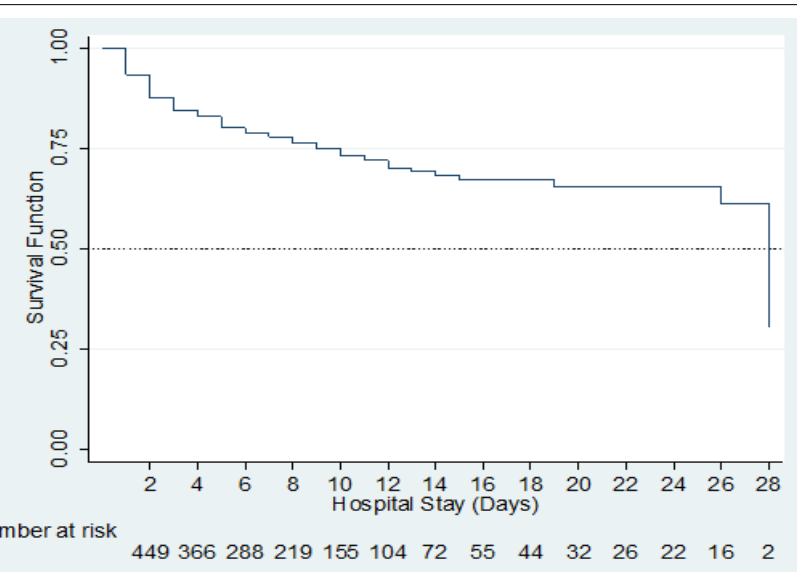

Figure 1: Kaplan Meier Estimate of Survival Function of Premature Infants.

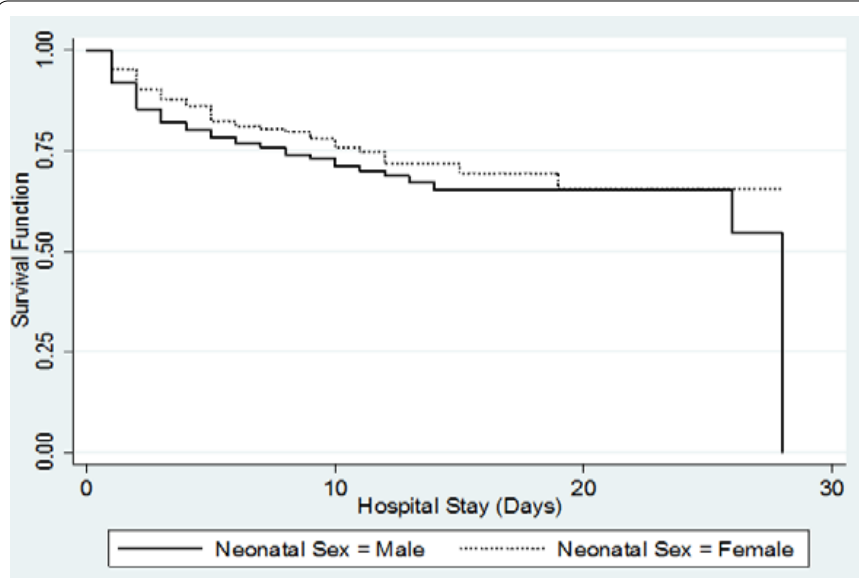

Figure 2: Comparison of KM curve of Neonatal Sex.

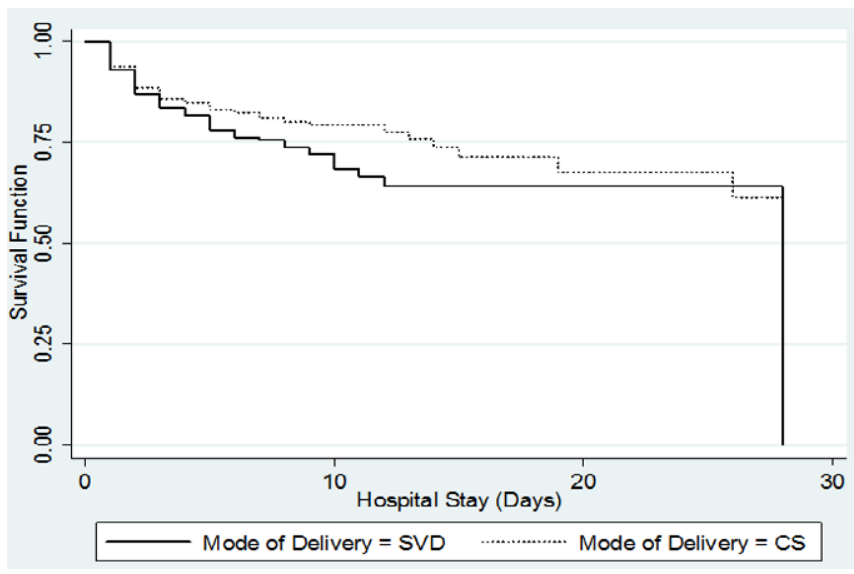

Figure 3: Comparison of KM curve of Mode of delivery.

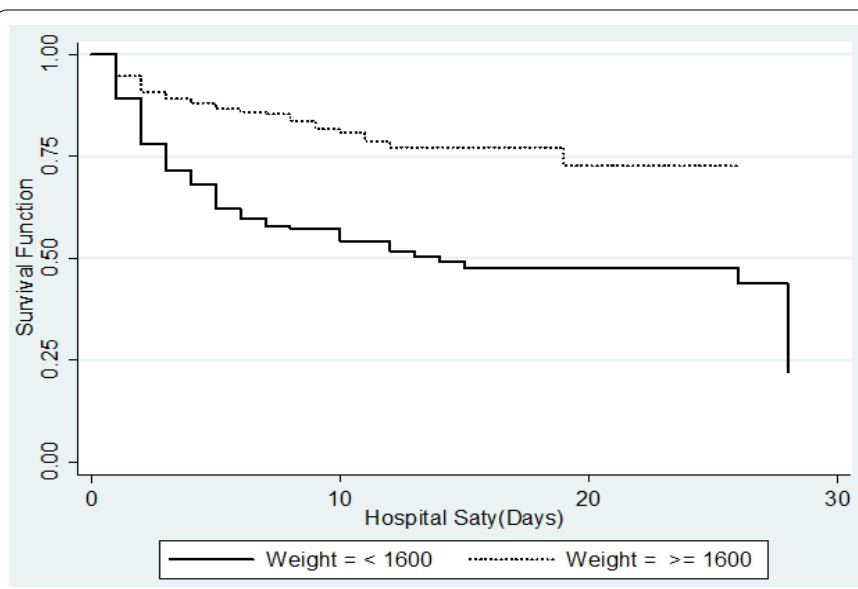

Figure 4: Comparison of KM curve of Weight of infants. 


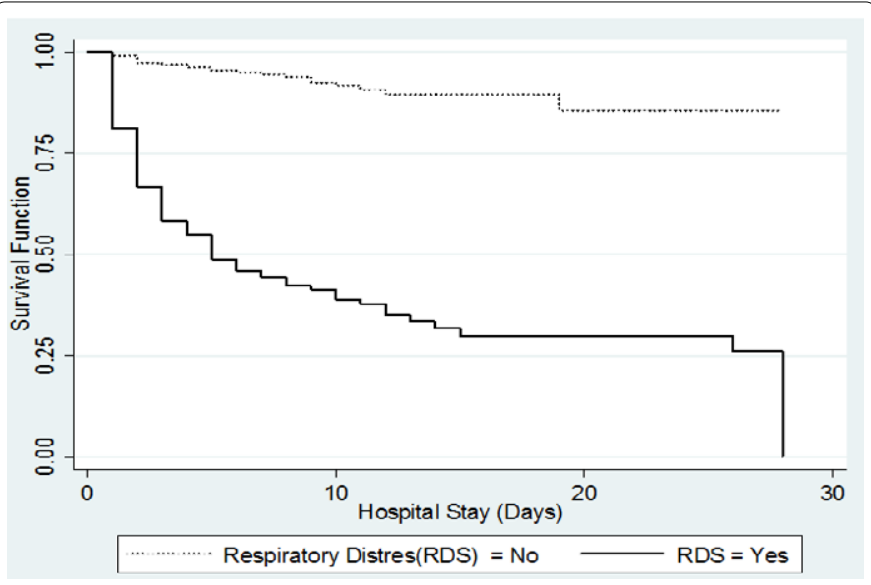

Figure 5: Comparison of KM curve of RDS.

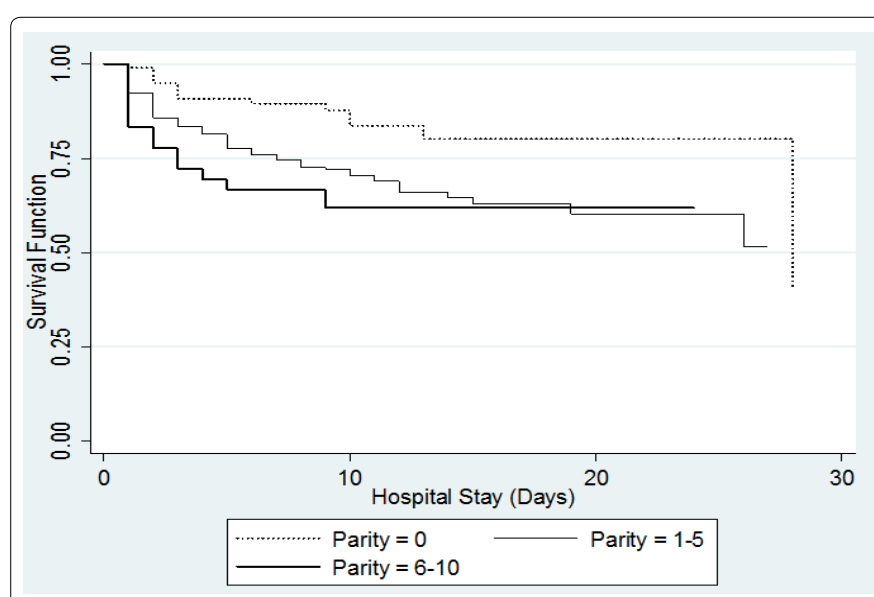

Figure 6: Comparison of KM curve of Parity

equivalent using the log-rank test. Therefore based on this statistical test procedure ANC, Gravidity, Parity, Weight of Infants, Gestational Age, Multiple Pregnancy, HIV status of Mother, IDM, Gestational Age $\mathrm{V}_{\mathrm{s}}$ Weight of infant, Breast Feeding Initiated, Hypothermia, Neonatal Sepsis, RDS, PNA, Anemia, Hypoglycemia, and Jaundice are statistically significant $(\mathrm{P}-$ Value $<0.05)$. This means that, we have enough evidence to say that the premature infants admitted to NICU survival curves are different or the Kaplan Meier curves are statistically different with respect to categories of significant covariates. Neonatal Sex, TTN, Congenital Malformation and Mode of delivery are not statistically significant ( $\mathrm{P}$-Value $>0.05$ ), that means, we have no enough evidence to say that the premature infants admitted to NICU survival curves are different or the Kaplan Meier curves are statistically equivalent with respect to categories of these covariates.

\section{Fitted cox proportional hazard model of premature infants}

The Cox proportional hazard model in Table 4 shows that ANC, Gravidity (6-10), HIV status, RDS, PNA, Anemia and Breast Feed Initiated were statistically significant $(\mathrm{P}$-Value $<0.05)$ determinants of premature infants mortality. However, other variables Mode of delivery, Gravidity (2-5), weight of infant, multiple pregnancies, AGA and LGA were not statistically significant.

The hazard ratio $(95 \% \mathrm{CI})$ for premature infants admitted to NICU who were born from those mothers had ANC, Gravidity of (6-10) and
HIV Positive, compared to those infants born from mothers hadn't ANC, gravidity of zero and HIV negative was 0.5247 [0.33828, 0.8139], $2.0717[1.00074,4.2889]$ and $1.8033[1.03724,3.1351]$, respectively. These means that, premature infants born from those mothers who had ANC follow up were $47.6 \%$ less likely to die than to those who hadn't ANC follow up. The risk of death of premature infants born from those mothers who had gravidity of (6-10) were 2.0717 times higher than to those infants born from mothers' gravidity of zero. Premature infants born from those mothers with HIV were $80.33 \%$ more likely to die than those infants born from mothers HIV negative.

The hazard ratio $(95 \% \mathrm{CI})$ for premature infants admitted to NICU who had RDS, PNA and anemia compared to those infants who hadn't RDS, PNA and anemia were 7.7742[4.71215, 12.826], 2.1248[1.41979, 3.1798] and 4.6699[1.7687, 12.3297], respectively. That is, the risk of death of premature infants those who had RDS, PNA and anemia were $7.7742,2.1248$ and 4.6699 times higher than to those infants without RDS, PNA and anemia respectively. The hazard ratio $(95 \%$ CI) for premature infants admitted to NICU those who were Breast feed initiated less than one, $[1,2]$ and greater than 2 hours compared to those who weren't breast feed initiated $0.1021[0.04480,0.2326]$, $0.1288[0.06760,0.2455]$ and $0.3752[0.22508,0.6254]$, respectively. Premature infants breast feed initiated less than one, $[1,2]$ and greater than 2 hours were $89.8 \%, 87.12 \%$ and $62.48 \%$ less likely to die than to those infants who weren't initiated at all, respectively.

\section{Fitted Semiparametric Gamma Frailty Model of Premature Infants}

It is argued that there is gestational age clustering in mortality among premature infants. We explored the effects of covariates on premature infants by accounting for categories of gestational age using semi parametric gamma frailty model.

The semi parametric gamma frailty model (Table 5) shows that ANC, Gravidity (6-10), HIV status, RDS, PNA, Anemia and Breast Feed Initiated were statistically significant $(\mathrm{P}$-Value $<0.05)$ determinants of premature infants' mortality within the same categories of premature infants by gestational age frailty effect. Mode of delivery, gravidity (25 ), weight of infant, multiple pregnancies, AGA and LGA were not statistically significant.

The hazard ratio $(95 \% \mathrm{CI})$ for premature infants admitted to NICU who were born from those mothers had ANC, gravidity of (6-10) and HIV positive, compared to those infants born from mothers hadn't ANC, gravidity of zero and HIV negative was $0.544(0.3475,0.853)$, $2.215(1.0597,4.629)$ and $1.776(1.0170,3.103)$, respectively. These means that, premature infants who were born from those mothers had ANC follow up were $45.6 \%$ less likely to die than to those who hadn't ANC follow up. The risk of death of premature infants that were born from those mothers had gravidity of (6-10) were 2.215 times higher than to those infants born from gravidity of zero mothers'. Premature infants that were born from mothers with HIV positive were $77.6 \%$ more likely to die than those infants born from mothers HIV negative.

The hazard ratio $(95 \% \mathrm{CI})$ for premature infants admitted to NICU who had RDS, PNA and anemia compared to those infants who hadn't RDS, PNA and anemia were 5.572(3.2655, 9.509), 1.916(1.2741, $2.882)$ and $7.053(2.5758,19.31)$, respectively. That is, the risk of death of premature infants those who had RDS and anemia were 5.572 and 7.053 times higher than to those infants without RDS and anemia, respectively. Premature infants who had PNA were $91.6 \%$ more likely to die than those infants without PNA. 
Citation: Yehuala S, Ayalew S, Teka Z (2015) Survival Analysis of Premature Infants Admitted to Neonatal Intensive Care Unit (NICU) in Northwest Ethiopia using Semi-Parametric Frailty Model. J Biomet Biostat 6: 223. doi:10.4172/2155-6180.1000223

\begin{tabular}{|c|c|c|c|c|}
\hline \multirow[b]{2}{*}{ Factors } & \multirow[b]{2}{*}{ Covariates } & \multicolumn{3}{|c|}{ Log-rank Test } \\
\hline & & DF & $x^{2}$ & P-Value \\
\hline & Neonatal Sex & 1 & 1.929 & 0.165 \\
\hline & Mode of delivery & 1 & 2.799 & 0.094 \\
\hline & Antenatal Care Visit(ANC) & 1 & 17.004 & $0.000^{* *}$ \\
\hline \multirow[t]{6}{*}{$\begin{array}{c}\text { Demographic and Obstetrics' } \\
\text { Factors }\end{array}$} & Gravidity & 2 & 11.607 & $0.003^{* *}$ \\
\hline & Parity & 2 & 12.8 & $0.002^{* *}$ \\
\hline & Weight of Infants & 1 & 34.031 & $0.000^{* *}$ \\
\hline & Gestational Age & 4 & 307.6 & $0.000^{* *}$ \\
\hline & Multiple Pregnancy & 1 & 12.097 & $0.001^{* *}$ \\
\hline & HIV status of Mother & 1 & 10.073 & $0.002^{* *}$ \\
\hline \multirow[t]{7}{*}{ Maternal Clinical Factors } & Infant of Diabetic Mother (IDM) & 1 & 9.124 & $0.003^{*+}$ \\
\hline & $\begin{array}{c}\text { Gestational Age Vs Weight of } \\
\text { infant }\end{array}$ & 2 & 13.329 & $0.001^{* *}$ \\
\hline & Breast Feeding Initiated & 4 & 266.178 & $0.000^{* *}$ \\
\hline & Hypothermia & 1 & 75.042 & $0.000^{* *}$ \\
\hline & Neonatal Sepsis & 1 & 42.681 & $0.000^{* *}$ \\
\hline & $\begin{array}{l}\text { Respiratory Distress Syndrome } \\
\text { (RDS) }\end{array}$ & 1 & 178.284 & $0.000^{* *}$ \\
\hline & Jaundice & 1 & 6.653 & $0.010^{* *}$ \\
\hline \multirow[t]{6}{*}{$\begin{array}{l}\text { Problems Identified at Birth } \\
\text { (Diagnosis of Infant) }\end{array}$} & Prinatal Asphyxia (PNA) & 1 & 64.481 & $0.000^{* *}$ \\
\hline & Anemia & 1 & 8.066 & $0.005^{* *}$ \\
\hline & Congenital Malformation & 1 & 1.446 & 0.229 \\
\hline & $\begin{array}{l}\text { Transient Tachypnea of } \\
\text { Newborn (TTN) }\end{array}$ & 1 & 3.018 & 0.082 \\
\hline & Hypoglycemia & 1 & 97.721 & $0.000^{* *}$ \\
\hline & Prematurity of Apnea & 1 & 130.935 & $0.000^{* *}$ \\
\hline
\end{tabular}

** Significant $(P$-value $<0.05)$

Table 3: Log-Rank test of equality of survival distributions of different factors of death of premature infants.

\begin{tabular}{|c|c|c|c|c|c|}
\hline Covariates & $\beta$ & $\operatorname{SE}(\beta)$ & $\mathbf{Z}$ & $\mathrm{HR}[95 \% \mathrm{Cl}]$ & $\operatorname{P}=\operatorname{Pr}(>|z|)$ \\
\hline \multicolumn{6}{|l|}{ Mode of Delivery $\quad$ (SVD) } \\
\hline CS & -0.2856 & 0.2172 & -1.315 & $0.7516[0.49101,1.1504]$ & 0.188535 \\
\hline \multicolumn{6}{|l|}{ ANC } \\
\hline Yes & -0.6449 & 0.224 & -2.879 & $0.5247[0.33828,0.8139]$ & $0.003987^{\star *}$ \\
\hline \multicolumn{6}{|l|}{ Gravidity } \\
\hline $2-5$ & 0.5514 & 0.2883 & 1.913 & $1.7358[0.98654,3.0539]$ & 0.055752 \\
\hline $6-10$ & 0.7284 & 0.3713 & 1.962 & $2.0717[1.00074,4.2889]$ & $0.049766^{* *}$ \\
\hline \multicolumn{6}{|l|}{ HIV status (Negative) } \\
\hline Positive & 0.5896 & 0.2822 & 2.09 & $1.8033[1.03724,3.1351]$ & $0.036658^{* *}$ \\
\hline \multicolumn{6}{|l|}{ Weight of Infant $\quad(<1600)$} \\
\hline$[1600,2500]$ & 0.1031 & 0.2109 & 0.489 & $1.1086[0.73320,1.6763]$ & 0.624978 \\
\hline \multicolumn{6}{|l|}{ Multiple Pregnancy (No) } \\
\hline Yes & 0.1289 & 0.2004 & 0.643 & $1.1376[0.76807,1.6849]$ & 0.520065 \\
\hline \multicolumn{6}{|l|}{ RDS $\quad$ (No) } \\
\hline Yes & 2.0508 & 0.2554 & 8.028 & $7.7742[4.71215,12.826]$ & $9.99 \mathrm{e}-16^{* *}$ \\
\hline \multicolumn{6}{|l|}{ PNA $\quad$ (No) } \\
\hline Yes & 0.7537 & 0.2057 & 3.664 & $2.1248[1.41979,3.1798]$ & $0.000248^{\star *}$ \\
\hline \multicolumn{6}{|l|}{ Anemia $\quad$ (No) } \\
\hline Yes & 1.5411 & 0.4954 & 3.111 & $4.6699[1.7687,12.3297]$ & $0.001863^{* *}$ \\
\hline \multicolumn{6}{|l|}{ GAvsWeight(SGA) } \\
\hline AGA & -0.2702 & 0.3209 & -0.842 & $0.7632[0.40689,1.4316]$ & 0.399816 \\
\hline LGA & -0.8544 & 1.067 & -0.801 & $0.4255[0.05256,3.4450]$ & 0.423275 \\
\hline \multicolumn{6}{|l|}{ Breast Feed Initiated (Not at all) } \\
\hline$<1 \mathrm{Hr}$ & -2.2821 & 0.4201 & -5.432 & $0.1021[0.04480,0.2326]$ & $5.58 \mathrm{e}-08^{* *}$ \\
\hline$[1,2] \mathrm{Hr}$ & -2.0493 & 0.329 & -6.228 & $0.1288[0.06760,0.2455]$ & $4.72 \mathrm{e}-10^{* *}$ \\
\hline$>2 \mathrm{Hr}$ & -0.9803 & 0.2607 & -3.76 & $0.3752[0.22508,0.6254]$ & $0.000170^{* *}$ \\
\hline Other Milk & -1.285 & 1.0364 & -1.24 & $0.2767[0.03628,2.1094]$ & 0.215046 \\
\hline
\end{tabular}

** Significant $(P$-value $<0.05)$ and categories in the bracket are reference categories.

Table 4: Final fitted Cox's Proportional Hazard Model of Premature infants' by stratification of hypothermia. 
Citation: Yehuala S, Ayalew S, Teka Z (2015) Survival Analysis of Premature Infants Admitted to Neonatal Intensive Care Unit (NICU) in Northwest Ethiopia using Semi-Parametric Frailty Model. J Biomet Biostat 6: 223. doi:10.4172/2155-6180.1000223

Page 8 of 12

\begin{tabular}{|c|c|c|c|c|c|c|}
\hline Covariates & Categories & $\beta$ & $\operatorname{SE}(\beta)$ & $x^{2}$ & $\mathrm{HR}[95 \% \mathrm{Cl}]$ & $\mathrm{p}$-value \\
\hline \multirow{2}{*}{ Mode of Delivery } & (SVD) & & & & & \\
\hline & CS & -0.283 & 0.218 & 1.69 & $0.753[0.4916,1.154]$ & $1.90 \mathrm{E}-01$ \\
\hline \multirow{2}{*}{ ANC } & (No) & & & & & \\
\hline & Yes & -0.608 & 0.229 & 7.05 & $0.544[0.3475,0.853]$ & $7.9 \mathrm{e}-03^{*}$ \\
\hline \multirow{3}{*}{ Gravidity } & -1 & & & & & \\
\hline & $5-\mathrm{Feb}$ & 0.543 & 0.286 & 3.61 & $1.720[0.9831,3.011]$ & $5.70 \mathrm{E}-02$ \\
\hline & 10-Jun & 0.795 & 0.376 & 4.47 & $2.215[1.0597,4.629]$ & $3.4 \mathrm{e}-02^{* \prime}$ \\
\hline \multirow{2}{*}{ HIV status } & (Negative) & & & & & \\
\hline & Positive & 0.575 & 0.285 & 4.08 & $1.776[1.0170,3.103]$ & $4.3 \mathrm{e}-02^{*}$ \\
\hline \multirow{2}{*}{ Weight of Infant } & $(<1600)$ & & & & & \\
\hline & {$[1600,2500]$} & 0.39 & 0.223 & 3.06 & $1.477[0.9543,2.285]$ & $8.00 \mathrm{E}-02$ \\
\hline \multirow{2}{*}{ Multiple Pregnancy } & (No) & & & & & \\
\hline & Yes & 0.117 & 0.202 & 0.34 & $1.124[0.7562,1.672]$ & $5.60 \mathrm{E}-01$ \\
\hline \multirow{2}{*}{ RDS } & (No) & & & & & \\
\hline & Yes & 1.718 & 0.273 & 39.69 & $5.572[3.2655,9.509]$ & $3.0 \mathrm{e}-10^{*}$ \\
\hline \multirow{2}{*}{ PNA } & (No) & & & & & \\
\hline & Yes & 0.65 & 0.208 & 9.75 & $1.916[1.2741,2.882]$ & $1.8 \mathrm{e}-03^{*}$ \\
\hline \multirow{2}{*}{ Anemia } & (No) & & & & & \\
\hline & Yes & 1.954 & 0.514 & 14.45 & $7.053[2.5758,19.31]$ & $1.4 \mathrm{e}-04^{* \prime}$ \\
\hline \multirow{3}{*}{ GAvsWeight } & (SGA) & & & & & \\
\hline & AGA & -0.266 & 0.321 & 0.68 & $0.767[0.4084,1.439]$ & 4.10E-01 \\
\hline & LGA & -0.766 & 1.072 & 0.51 & $0.465[0.0568,3.801]$ & $4.70 \mathrm{E}-01$ \\
\hline \multirow{5}{*}{ Breast Feed Initiated } & (Not at all) & & & & & \\
\hline & $<1 \mathrm{Hr}$ & -2.214 & 0.444 & 24.91 & $0.109[0.0458,0.261]$ & $6.0 \mathrm{e}-07^{* \prime}$ \\
\hline & {$[1,2] \mathrm{Hr}$} & -1.824 & 0.345 & 27.91 & $0.161[0.0820,0.317]$ & $1.3 e-07^{* \prime}$ \\
\hline & $>2 \mathrm{Hr}$ & -0.948 & 0.269 & 12.39 & $0.388[0.2287,0.657]$ & $4.3 \mathrm{e}-04^{* \prime}$ \\
\hline & Other Milk & -1.766 & 1.07 & 2.72 & $0.171[0.0210,1.393]$ & $9.90 \mathrm{E}-02$ \\
\hline \multicolumn{4}{|c|}{ Frailty(Gestational Age, distribution= "Gamma") } & 13.91 & & $3.1 \mathrm{e}-03^{* \prime}$ \\
\hline \multicolumn{4}{|c|}{ Variance of Random Effect $=\operatorname{Var}(U)=\theta$} & & 0.252 & \\
\hline
\end{tabular}

** Significant $(P$-value $<0.05)$ and categories in the bracket are reference categories.

Table 5: Final fitted Semiparametric Gamma frailty Model of Premature infants' by stratification of hypothermia.

Finally, the hazard ratio $(95 \% \mathrm{CI})$ for premature infants admitted to NICU had Breast feed initiated less than one, [1,2] and greater than 2 hours compared to those who hadn't breast feed initiated was $0.109(0.0458,0.261), 0.161(0.0820,0.317)$ and $0.388(0.2287,0.657)$, respectively. Premature infants had breast feed initiated less than one, $[1,2]$ and greater than 2 hours were $89.1 \%, 83.9 \%$ and $61.2 \%$ less likely to die than to those infants who hadn't initiated at all, respectively.

Table 5 shows that the variance of frailty is significantly greater than zero $(\theta=0.252$, P-Value $=0.0031<\alpha=0.05)$. It shows that each category of preterm infants by gestational age has different values of random effects and there is heterogeneity of risks between gestational age categories.

In empirical applications, the observed survival data are used to estimate the parameters of the distribution of frailty $f(u)$ i.e. in this study gamma distribution and to actually predict the individual frailties $\left(U_{j}\right)$. Premature infants in the same categories of preterm based on gestational age usually share the same unobserved frailty.

From Table $6 U_{j}$ is the common frailty which is shared by all premature infants' in group $\mathrm{j}$ and we call it as shared frailty. $U_{j}$ is a measure of relative risk because the greater an individual's frail(Hanagal, 2011); with regard to premature infants' death, the greater the infants' susceptibility to the premature infants' death.

Table 6 shows the common frailty $\mathrm{U}_{j}(95 \% \mathrm{CI})$ of each premature infant in categories of preterm based on gestational age $(\leq 28),(28$, $30]$ and $(30,32]$ is $1.575(0.9207,2.695), 1.254(0.6325,2.487)$ and
$1.135(0.6568,1.960)$, respectively. Premature infants in these groups are more frail infants $\left(U_{j}>1\right)$, i.e. they have a greater hazard and more likely to die earlier. However, the common frailty $\mathrm{U}_{j}(95 \% \mathrm{CI})$ for categories $(32,34]$ and $(34,37)$ is $0.527[0.2646,1.049]$ and $0.509[0.2357,1.099]$, respectively and infants in these groups are less frail infants $\left(U_{j}<1\right)$, they have a smaller hazard and are less likely to die earlier.

Generally, the greater the common frailty effect $\left(U_{j}\right)$ the more likely to get the event (death)((Hanagal, 2011)). Therefore, infants in gestational age group $(\leq 28)$ are more likely to die than other groups whereas; premature infants in gestational age group $(34,37)$ are less likely to die than other group.

\section{Checking the adequacy of fitted premature infants models}

The final Cox proportional hazard and semi parametric gamma frailty models were fitted after both the outliers, overall goodness of test and proportional hazard assumptions are fulfilled. The other aspects of the final models examined were the influence or leverage each subject has on the model fit. The following sections show the test results for overall fit and proportional hazard assumptions of final fitted premature infants' models.

\section{Testing the overall fit of premature infants models}

The overall fit for Cox proportional hazard and semi parametric gamma frailty models was tested by likelihood ratio and Wald test, as shown in Table 7. Results from these test procedures; we have enough evidence to say that both models are best fit $(\mathrm{P}$-Value $<0.05)$ at $5 \%$ level 


\begin{tabular}{|l|l|}
\hline Group=j & $\mathbf{U}_{\mathbf{j}}=\exp \left(\mathbf{w}_{\mathbf{j}} \mathbf{\phi}\right)[\mathbf{9 5} \% \mathbf{C l}]$ \\
\hline Gamma: gestational Age $(\leq 28)$ & $1.575[0.9207,2.695]$ \\
\hline Gamma: gestational Age $(28,30]$ & $1.254[0.6325,2.487]$ \\
\hline Gamma: gestational Age $(30,32]$ & $1.135[0.6568,1.960]$ \\
\hline Gamma: gestational Age $(32,34]$ & $0.527[0.2646,1.049]$ \\
\hline Gamma: gestational Age $(34,37)$ & $0.509[0.2357,1.099]$ \\
\hline
\end{tabular}

Table 6: Common frailty term of premature infants in categories of preterm by gestational age.

of significance. A perfectly adequate model may have low $\mathrm{R}^{2}$ due to high percent of censored data, but the concordance probability which is used to evaluate the discriminatory power and the predictive accuracy of the two models is large.

\section{Testing the Validity of Proportional Hazard Assumption}

Testing the proportional assumption is vital for interpretation and use of fitted proportional hazard and semi parametric proportional hazard models. Therefore, in this study the Schoenfeld residuals proportional hazard assumption test for the individual covariates used., while the global test uses the scaled Schoenfeld residuals by R-software cox.zph code. If significant ( $\mathrm{P}-\mathrm{V}$ alue $<0.05)$, then the proportional hazard assumption is rejected. From Table 8 , we observe that each covariates (P-Value $>0.05$ ) and all of covariates simultaneously (GLOBAL for Cox proportional hazard P-Value $=0.9334>0.05$ and semi parametric proportional hazard $\mathrm{P}-$ Value $=0.8861$ ) met the proportional hazard assumption.

\section{Comparison of premature infants models}

The AIC, BIC, and Log-likelihood (full) are used for comparing the Cox proportional hazard and semi parametric gamma frailty models. The semi parametric gamma frailty model was considered the best model as it had the smallest AIC, BIC and I-likelihood (full) compared to the Cox proportional hazard model (Table 9).

In addition to these, $R^{2}$ and concordance are used for model comparison. A perfectly adequate model may have low $\mathrm{R}^{2}$ due to high percent of censored data for both models [27,28], but $R^{2}$ for semi parametric gamma frailty is greater than Cox proportional hazard model. The concordance probability is used to evaluate the discriminatory power and the predictive accuracy of statistical models. In this study, the predictive accuracy of semi parametric gamma frailty model is greater than Cox proportional hazard model.

\section{Discussion of the results}

This study shows that the overall mortality of premature infants admitted to NICU in UoGH during the study period was $122(25.2 \%)$ and that was very satisfactory in comparison to the study carried out by Fakher et al., [29], 48\% death of preterm infants admitted to NICU in Fawzy Moaz Hospital, Egypt. However, more serious as compared to the study $20 \%$ death of preterm infants in developed countries (All European and Asian). The overall mean and median survival time of premature infants admitted to NICU of UoGH were 20.38 and 28 days, respectively.

The log-rank test and Kaplan Meier curve for equality of survival distribution of categories of covariates shows that ANC, Gravidity, Parity, Weight of Infants, Gestational Age, Multiple Pregnancy, HIV status of Mother, IDM, Gestational Age Vs Weight of infant, Breast Feeding Initiated, Hypothermia, Neonatal Sepsis, RDS, PNA, Anemia, Hypoglycemia, and Jaundice are statistically significant (P-Value $<0.05$ ). The study shows that, we have enough evidence to say that the premature infants admitted to NICU survival distribution are different or the Kaplan Meier curves are statistically different with respect to categories of significant covariates. This finding is consistent with the study done by [30], in France for SGA and multiple pregnancies (birth). Other variables like Neonatal Sex, TTN, Congenital Malformation and Mode of delivery are not statistically significant (P-Value $>0.05)$, that means, we have no enough evidence to say that the premature infants admitted to NICU survival curves are different or the Kaplan Meier curves are statistically equivalent with respect to categories of these covariates and not consistent with the study done by [30] in France for neonatal sex.

In this study, we well fitted the Cox proportional hazard and semi parametric gamma frailty models to estimate the hazard of premature infants. We identified the potential determinant of premature infants' mortality by applying the stepwise selection of covariates for both models. Based on this variable selection procedure; mode of delivery, ANC, Gravidity, HIV status, weight of infant, multiple pregnancy, hypothermia, RDS, PNA, anemia, GA Vs Weight and breast feed initiated are included in the final analysis of the two models. However, hypothermia did not satisfy the proportional assumption. Therefore, Cox proportional hazard and semi parametric gamma frailty models stratified by hypothermia were fitted as suggested by [31]. Among these variables ANC similar to the studies done by $[32,33]$, gravidity of (610), HIV status of mother consistent to the study done by [34], RDS similar to the studies done by $[4,33]$, PNA similar to the study done by [4], anemia and breastfeed initiated were the most determinant and statistically significant variables for mortality of premature infants in both Cox proportional hazard and semi parametric frailty models. These significant variables are statistically associated with time to death of premature infants admitted to NICU. These finding is consistent with the studies done by [4,32-34] for ANC, RDS, HIV status and PNA.

Both log-likelihood, AIC, BIC, R2 and concordance model comparison methods were applied. Semiparametric gamma frailty model was considered the best model as it had the highest R2 and concordance; and lowest I-likelihood, AIC and BIC compared to the Cox proportional hazard model which is similar to a study carried out by Mani et al., for modeling under five children, [17], for breast cancer and [19] for time to malaria infection data sets.

There was frailty effect $(\theta=0.252$, P-Value $=0.0031, \alpha=0.05)$ and mortality were dependent within as well as across categories of preterm infants based on their gestational age. The values of frailty were dispersed and hence induced greater heterogeneity in the infant hazards. It also indicates stronger association within categories of premature infants. Chances of survival among these infants vary greatly according to the length of gestation. At the lowest gestational ages, a large proportion of deaths with the poorest survival prognosis.

Premature infants that were born from mothers who had ANC follow up were statistically significant determinant for mortality of premature infants admitted to NICU. The study done by Turmen and motherhood [35], states that adequate ANC was recognized as an important factor in the reduction of maternal and newborn deaths. So the purpose of ANC is to decrease the number of infants born too soon (premature birth), too small (low birth weight) and to prevent mother and infant sickness and death [36].

Premature infants those were born from mothers with HIV positive were $77.6 \%$ more likely to die than those infants born from mothers HIV negative. This finding is consistent with the study done by Slyker et al. Preterm labour may be more common in HIV positive women, with rates as high as double those rates seen in uninfected women in some reports $[37,38]$. 
Citation: Yehuala S, Ayalew S, Teka Z (2015) Survival Analysis of Premature Infants Admitted to Neonatal Intensive Care Unit (NICU) in Northwest Ethiopia using Semi-Parametric Frailty Model. J Biomet Biostat 6: 223. doi:10.4172/2155-6180.1000223

Page 10 of 12

\begin{tabular}{|c|c|c|c|c|c|}
\hline & & Likelihood Ratio test statistic & Wald test statistic & & \\
\hline & & (P-Value) & (P-Value) & $\mathbf{R}^{2}$ & \\
\hline Models & p & & & (Max.Possible) & Concordance \\
\hline Cox Proportional Hazard & 16 & $264.3(0.000)$ & $181.3(0.000)$ & $0.420(0.923)$ & 0.891 \\
\hline Semiparametric Gamma Frailty & 19 & $283.0(0.000)$ & $185.0(0.000)$ & $0.442(0.923)$ & 0.915 \\
\hline
\end{tabular}

Where: $P=$ Number of parameter estimated

Table 7: Testing the Overall Fit of Cox Proportional Hazard and Semiparametric Gamma Frailty Models.

\begin{tabular}{|c|c|c|c|c|c|c|}
\hline \multirow[b]{2}{*}{ Covariates } & \multicolumn{3}{|c|}{ Cox-Proportional Hazard } & \multicolumn{3}{|c|}{ Semiparametric Gamma Frailty } \\
\hline & $\rho$ (rho) & $x^{2}$ & p-value & $\rho$ (rho) & $\mathrm{x}^{2}$ & p-value \\
\hline \multicolumn{7}{|l|}{ Mode of Delivery $\quad$ (SVD) } \\
\hline CS & -0.139721 & $3.21 \mathrm{E}+00$ & 0.0733 & -0.1564 & $4.05 \mathrm{E}+00$ & 0.05141 \\
\hline \multicolumn{7}{|l|}{ ANC $\quad$ (No) } \\
\hline Yes & -0.041893 & $2.51 \mathrm{E}-01$ & 0.6165 & 0.0024 & 8.54E-04 & 0.9767 \\
\hline \multicolumn{7}{|l|}{ Gravidity } \\
\hline $2-5$ & -0.004627 & 2.79E-03 & 0.9579 & -0.0103 & $1.32 \mathrm{E}-02$ & 0.9085 \\
\hline $6-10$ & -0.069094 & $6.22 \mathrm{E}-01$ & 0.4302 & -0.0317 & $1.35 \mathrm{E}-01$ & 0.7128 \\
\hline \multicolumn{7}{|l|}{ HIV status(No) } \\
\hline Yes & 0.065262 & $6.05 \mathrm{E}-01$ & 0.4366 & 0.073 & $7.40 \mathrm{E}-01$ & 0.3898 \\
\hline \multicolumn{7}{|l|}{ Weight of Infant $\quad(<1600)$} \\
\hline$[1600,2500]$ & -0.03918 & $2.70 \mathrm{E}-01$ & 0.6034 & -0.0181 & $5.10 \mathrm{E}-02$ & 0.8214 \\
\hline \multicolumn{7}{|l|}{ Multiple Pregnancy (No) } \\
\hline Yes & -0.018613 & 4.39E-02 & 0.8341 & 0.022 & 6.14E-02 & 0.8042 \\
\hline \multicolumn{7}{|l|}{ RDS $\quad$ (No) } \\
\hline Yes & 0.014764 & 3.36E-02 & 0.8545 & -0.0177 & 5.34E-02 & 0.8172 \\
\hline \multicolumn{7}{|l|}{ PNA $\quad$ (No) } \\
\hline Yes & -0.005023 & $3.49 \mathrm{E}-03$ & 0.9529 & -0.0211 & $5.94 \mathrm{E}-02$ & 0.8075 \\
\hline \multicolumn{7}{|l|}{ Anemia $\quad$ (No) } \\
\hline Yes & -0.009122 & 1.11E-02 & 0.9161 & 0.0299 & $1.11 \mathrm{E}-01$ & 0.7386 \\
\hline \multicolumn{7}{|l|}{ GAvsWeight(SGA) } \\
\hline AGA & 0.029036 & $1.23 \mathrm{E}-01$ & 0.7256 & 0.0118 & $1.98 \mathrm{E}-02$ & 0.8881 \\
\hline LGA & 0.069587 & 5.99E-01 & 0.4388 & 0.0569 & 4.00E-01 & 0.527 \\
\hline \multicolumn{7}{|l|}{ Breast Feed Initiated(Not atall) } \\
\hline$<1 \mathrm{Hr}$ & -0.000616 & 5.09E-05 & 0.9943 & 0.0388 & $2.11 \mathrm{E}-01$ & 0.6462 \\
\hline$[1,2] \mathrm{Hr}$ & 0.106853 & $1.37 \mathrm{E}+00$ & 0.2425 & 0.1509 & $2.97 \mathrm{E}+00$ & 0.0847 \\
\hline$>2 \mathrm{Hr}$ & 0.044064 & $2.43 E-01$ & 0.6221 & 0.0934 & $1.11 \mathrm{E}+00$ & 0.2917 \\
\hline Other Milk & 0.02515 & 7.54E-02 & 0.7836 & 0.0244 & 7.57E-02 & 0.7831 \\
\hline GLOBAL & NA & $8.48 \mathrm{E}+00$ & 0.9334 & NA & $1.36 \mathrm{E}+01$ & 0.8861 \\
\hline
\end{tabular}

Categories in the bracket are reference categories.

Table 8: Schoenfeld Residuals test for proportionality assumption of each covariate and overall model of the stratified Cox Proportional Hazard and Semiparametric Gamma Frailty Models.

Breast feed initiated less than one, [1,2] and greater than 2 hours were statistically significant determinant for mortality of premature infants admitted to NICU. Significantly positive effects of human milk feeding on long-term neurodevelopment are observed in preterm infants [39].

Other covariates such as mode of delivery, weight of infant, multiple pregnancy and GA Vs Weight were not statistically significant, suggesting that these variables are not associated with time to death of premature infants admitted to NICU in both models. However, these variables were statistically significant for mortality of premature infants in studies $[32,33,40]$.

\section{Conclusion}

Based on the results of log-rank test and Kaplan Meier curve for equality of survival distributions between categories of covariates; we can conclude that survival prognosis among premature infants was lower for mothers hadn't ANC, greater than one Gravidity, greater than zero Parity, Weight of Infants less than 1600 gram, Gestational Age less than 28 weeks, twins (multiple pregnancy), HIV positive of mother, IDM, SGA, no breast feeding initiated, Hypothermia, Neonatal Sepsis, RDS, PNA, Anemia, Hypoglycemia, and Jaundice. Whereas, the survival distributions between categories of other variables like Neonatal Sex, TTN, Congenital Malformation and Mode of delivery were statistically the same.

Both Cox proportional hazard and semi parametric frailty models identifies ANC, gravidity of (6-10), HIV status of mother, RDS, PNA, anemia and breastfeed initiated as the most determinant and statistically associated with time to death of premature infants admitted to NICU. We have compared the models using log-likelihood, AIC, $\mathrm{BIC}, \mathrm{DIC}, \mathrm{R}^{2}$ and concordance. From the value of these entire criterion; we conclude that the semi parametric gamma frailty model provide a suitable estimation of parameters for premature infants data as 
Citation: Yehuala S, Ayalew S, Teka Z (2015) Survival Analysis of Premature Infants Admitted to Neonatal Intensive Care Unit (NICU) in Northwest Ethiopia using Semi-Parametric Frailty Model. J Biomet Biostat 6: 223. doi:10.4172/2155-6180.1000223

Page 11 of 12

\begin{tabular}{|l|c|c|c|c|c|c|}
\hline & & I-likelihood & I-likelihood & & \\
\hline Models & p & (Null) & (Full) & AIC & BIC & $\boldsymbol{R}^{\mathbf{2}}$ \\
\hline Cox Proportional Hazard & 16 & -622.0952 & -489.9576 & 1011.915 & 1078.862 & 0.42 \\
\hline Semiparametric Gamma Frailty & 19 & -622.0952 & -480.4515 & 998.903 & 1077.307 & 0.891 \\
\hline
\end{tabular}

Where: $P=$ Number of parameter estimated

Table 9: Log-Likelihood, AIC and BIC of Cox Proportional Hazard and Semiparametric Gamma Frailty Models.

compared to Cox proportional hazard model. There was heterogeneity or frailty effect in categories of premature infants based on gestational age. Therefore, premature infants in the same categories share the same unobserved frailty.

From the study, we recommend that more efforts should be exerted to evaluate the magnitude of the problem of prematurity in UoGH through better coverage of antenatal care in the primary health care centers and through better statistical systems in both primary care and hospitals. The medical managements better to arrange a program for continuous training to the medical staff for better assessment, diagnosis and management of premature cases. The potent benefits of human milk are such that all preterm infants should receive human milk. Milk from the infant's own mother, fresh or previously frozen, should be the primary diet, and it should be fortified appropriately for the infants. If the mother's milk is unavailable despite significant lactation support, pasteurized donor milk should be used. There medical managements should facilitate more research to find out more precise diagnosis of causes of premature infants and maternal adverse outcome with better computerized recording system.

\section{References}

1. World Health Organisation (2012) Born Too Soon: The Global Action Report On Preterm Birth. In: Howson, Kinney M. \& Lawn E. (Eds.). Geneva.

2. Offiah I, O'Donoghue K, Kenny L (2012) Clinical Risk Factors for Preterm Birth 3: 74-91.

3. Santa EJ, Cox T (2006) Preterm Birth: Causes, Consequences, and Prevention.

4. Rehana T (2006) Preterm Delivery A Major Predictor of Perinatal Morbidity and Mortality. Jpm 20: 279-283.

5. Lawn JE, Gravett MG, Nunes T, Rubens E, Stanton C, et al. (2010) Global Report on Preterm Birth and Stillbirth (1 of 7): Defi Nitions, Description of the Burden and Opportunities to Improve Data. Bmc Pregnancy and Childhood 10: 1-22.

6. Howson CP, Kinney MV, Mcdougall L (2013) Born Too Soon: Preterm Birth Matters. Reproductive Health Journal 10: 1-9.

7. Blencowe H, Cousens S, Oestergaard MZ, Chou D, Moller AB, et al. (2012) National, Regional, And Worldwide Estimates Of Preterm Birth Rates In The Year 2010 With Time Trends Since 1990 For Selected Countries: A Systematic Analysis and Implications. The Lancet 379: 2162-2172.

8. UNICEF (2012) Maternal, Newborn and Child Survival, Country Profile, Ethiopia. Statistics and Monitoring Section / Policy and Practice.

9. Risso SDP, Nascimento LFC (2010) Risk Factors for Neonatal Death in Neonatal Intensive Care Unit According to Survival Analysis. Universidade Detaubate Unitau Taubaté (Sp) Brazil 22: 9-26.

10. Clayton DG (1978) A Model For Association In Bivariate Life Tables And Its Application In Epidemiological Studies of Familial Tendency in Chronic Disease Incidence. Biometrika, 65: 141-151.

11. Hougaard $P$ (2000) Analysis Of Multivariate Survival Data. Springer, Newyork, $1,255-273$.

12. Abdulkarimova U (2013) Frailty Models For Modelling Heterogeneity. Digitalcommons at Mcmaster Open Access Dissertations and Theses 1: 1-75.

13. Aalen OO (1994) Effects of Frailty in Survival Analysis. Statistical Methods in Medical Research. Biometrics 3: 227-243.

14. Lancaster T (1979) Econometric Methods For The Duration Of Unemployment Econometrica 47: 439-454.
15. Vaupel JW, Manton KG, Stallard E (1979) The Impact Of Heterogeneity in Individual Frailty On The Dynamics Of Mortality. Demography 16: 439-454.

16. Mani K, SN Dwivedi, P Pandey RM (2012) Determinants Of Under-Five Mortality In Rural Empowered Action Group States In India: An Application Of Cox Frailty Model. International Journal Of Mch And Aids 1: 60-72.

17. Faradmal, J, Talebi A, Rezaianzadeh A, Mahjub H (2012) Survival Analysis of Breast Cancer Patients Using Cox and Frailty Models. Journal of Research in Health Sciences (Jrhs) 12: 127-130.

18. Wang N (2011) Hierarchical Likelihood Approach for the Weibull Frailty Model Journal of Statistical Computation And Simulation 81: 343-356.

19. Belay B, Yehenew G, Delenasaw Y (2011) Modeling Time-to-Malaria Comparision of Cox and Frailty Model A Case of Under 10 Children Around Gilgel Gibe Hydroelectric Dam Ethiopia. Lambert.

20. Kadane J, Lazar N (2001) Methods and Criteria for Model Selection. Technical Report. Carnegie Mellon University.

21. Rao C, Wu Y (2001) On Model Selection (With Discussion) In P. Lahiri (Ed.), Model Selection. Technical Report 38: 1-57.

22. Yan YF (2011) Survival Analysis Final Report: Data Analysis For The Sequentia Primary Biliary Cirrhosis Data. Computational Statistics and Data Analysis 53: 2605-2616.

23. Akaike H (1973) Information Theory and an Extension of the Maximum Likelihood Principle: Second International Symposium on Information Theory. Eds. B. N. Petrox and F. Caski, Budapest: Akademiai Kiado 267-281.

24. Schwarz GE (1978) Estimating the Dimension of a Model. Annals of Statistics 6: 461-464.

25. Shibata R (1976) Selection of the order of an Autoregressive Model by Akaikes Information Criterion. Biometrika 63: 117-126.

26. Katz RW (1981) On Some Criteria for Estimating the Order of a Markov Chain Technometrics 23: 243-249.

27. Collett D (1994) Modelling Survival Data in Medical Reseach. ( $2^{\text {nd }}$ Edition), In Collett, D. (Ed.) Text In Statistical Science. London: Chapman and Hall/Crc.

28. Hosmer DJ, Lemeshow S (1999) Applied Survival Analysis: Regression Modeling of Time to Event Data. ( $2^{\text {nd }}$ Edition), John Wiley \& Sons, Inc, Canada.

29. Fakher M, Shaaban W, Monein AA, Hassan Z, Fikry MM (2005) Statistical Study Of Preterm Infants Admitted To NICU In Fawzy Moaz 19: 155-158.

30. Larroque B, Breart G, Kaminski M, Dehan M, Andre M, et al. (2004) Survival of Very Preterm Infants: Epipage, A Population Based Cohort Study. Archives of Disease in Childhood. Fetal and Neonatal Edition, 89: f139-f144.

31. Kleinbaum D, Klein M (2005) Survival Analysis A Self-Learning Text. (3r Edition), Springer.

32. Bader D, Kugelman A, Boyko V, Levitzki O, Lerner-Geva L, et al. (2010) Risk Factors and Estimation Tool for Death among Extremely Premature Infants: A National Study. Pediatrics 125: 696-703.

33. Sonkusare S, Rai L, Naik P (2009) Preterm Birth: Mode of Delivery and Neonatal Outcome. Medical Journal 64: 303-306.

34. Slyker JA, Patterson J, Ambler G, Richardson BA, Obimbo E, et al. (2014) Correlates and Outcomes of Preterm Birth, Low Birth Weight, and Small for Gestational Age in Hiv-Exposed Uninfected Infants. Bmc Pregnancy and Childhood 14: 2393-2397.

35. Turmen T, Motherhood S (1998) Antenatal Care. Report of Technical Working Group. WHO Geneva. Eastern Mediterranean Health Journal 4.

36. Ziyo FY, Matly FA, Mehemd GM, Dofany EM (2009) Relation between Prenata Care and Pregnancy Outcome At Benghazi. Sudanese Journal of Public Health 4: 403-410. 
Citation: Yehuala S, Ayalew S, Teka Z (2015) Survival Analysis of Premature Infants Admitted to Neonatal Intensive Care Unit (NICU) in Northwest Ethiopia using Semi-Parametric Frailty Model. J Biomet Biostat 6: 223. doi:10.4172/2155-6180.1000223

Page 12 of 12

37. Miotti P, Chiphangwi J, Dallabetta G (1992) The Situation in Africa. Ballieres Clinical Obstet Gynecology. 6: 165-185

38. Mcintyre J (1994) Pregnancy and HIV Infection at Baragwanath Hospital 6 $165-185$.
39. Eidelman, AIM, Schanler RJM (2012) Breastfeeding and the use of Human Milk. The American Academy of Pediatrics 129.

40. Turker G, Doger E, Arısoy E, Günlemez A, Gökalp S (2013) The Effect of IVF Pregnancies on Mortality and Morbidity in Tertiary Unit. Italian Journal of Pediatrics 39: 17. 